\title{
On-line Monitoring of Ethanol Concentration during Biomass Fermentation.
}

\author{
Ranran Zhang \\ College of Electrical Engineering and Automation, Qilu University of Technology (SDAS), Jinan \\ 250353, China
}

Keywords: neural network; the fermentation.

\begin{abstract}
For fermentation process large time delay, nonlinear and distributed parameter and disturbance, to combine neural networks and fuzzy control, neural network has the ability to learn, to modify control rules, the proposed neural network controller, realize online monitoring of biomass in the fermentation process of ethanol concentration, to accelerate the learning speed, through the fermentation process control, shows that the control performance is better than that of general fuzzy control, and wide application scope.
\end{abstract}

\section{Introduction}

Biomass in the fermentation process is the key process parameters, due to the limitation of biological sensing technology development level, has not well solve the problem of online measuring the biomass, such as ethanol concentration online monitoring cannot be achieved, the traditional fermentation control system is generally in the fermentation process on the basis of the basic physical quantities for testing $[1,2]$. The control cabinet supplied by the manufacturer is usually composed of four parameters: temperature, flow rate, $\mathrm{PH}$ value, and DO [3]. The feeding material needs to be added by hand in batches. In the process of fermentation, it is necessary to take samples to measure various chemical parameters and biological parameters during the time period, and there are serious nonlinear and uncertain problems [4].

On the basis of the traditional method to establish soft sensor model is difficult to real description of the process characteristics, and using the neural network method cannot need to be aware of the prior knowledge of the steady-state and dynamic process under the condition of very convenient soft measurement model is established, and as the change of industrial process internal characteristics, model can learn to be fixed in time, this makes the neural network soft measurement and inference control of the main tools.

\section{Existing Detection Methods of Ethanol.}

In general, there are many convenient laboratory techniques to detect the content of ethanol, for example: gas chromatography, high performance liquid chromatography (HPLC) method, the head space GC FID, enzymatic method, chemical analysis, Raman spectrum analysis method [5] and so on. The table 1 is the record table for the change of alcohol concentration with time. Figure 1 is an alcohol time curve.

Table 1. Alcohol concentration record table for experimental process

\begin{tabular}{|c|c|c|c|c|c|c|c|}
\hline \multirow{2}{*}{ Detection project } & \multicolumn{7}{|c|}{ Fermentation time(h) } \\
\cline { 2 - 8 } & 0 & 6 & 21 & 29 & 43 & 49 & 54 \\
\hline Alcohol content $\left(\%, 20^{\circ} \mathrm{C}\right)$ & & & 1 & 2.5 & 4.2 & 4.9 & 5.7 \\
\hline PH value of fermented mash & 4.98 & 4.36 & 3.95 & 3.85 & 3.43 & 3.35 & 3.28 \\
\hline
\end{tabular}




\section{Alcohol degree - time}

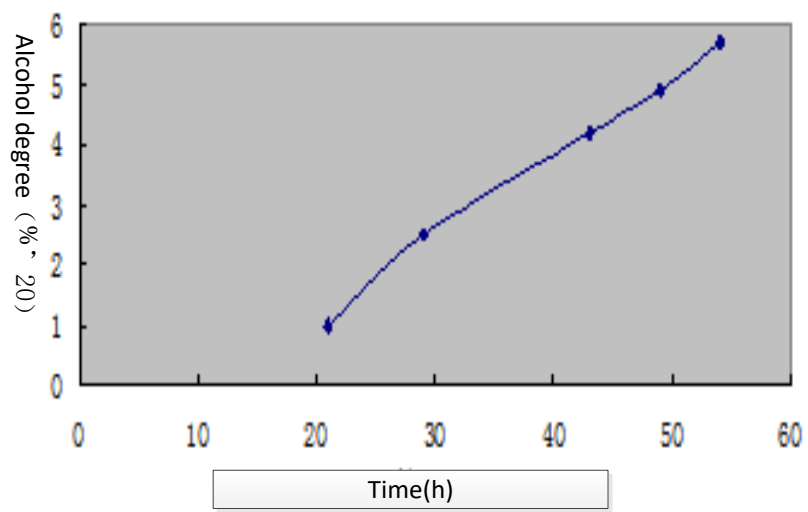

Fig.1 Alcohol-time

\subsection{Gas Chromatography.}

Gas chromatography has a wide range of applications. It has been established as one of the national standards for measuring the accuracy of wine, wine and beer

\subsection{Alcohol Meter Method}

The current national hygienic standard for alcohol and distilled spirits is determined by alcohol method. Alcohol content is widely used in the determination of alcohol content.

\subsection{Density Bottle Method}

The density bottle method is used to accurately determine the sample of alcohol precision, but the temperature change seriously affects the determination result, and the operation is tedious and takes a long time.

\subsection{Iodometric Titration.}

Iodometric titration is used to determine ethanol in vegetables, distillation column wastewater and alcohol.

\section{Establishment of Soft Measurement Model of Artificial Neural Network.}

\subsection{The Basic Structure of the Soft Measurement Model is Shown in Figure 1.}

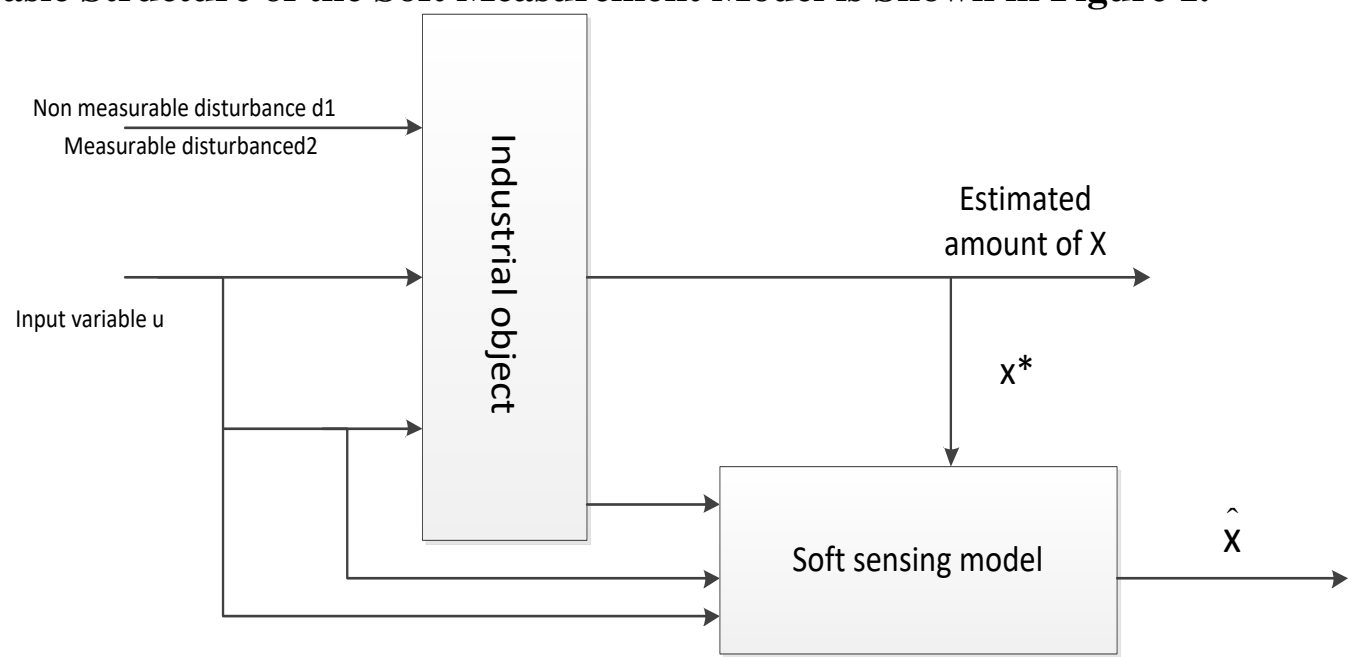

Fig. 2 The basic structure of the soft measurement model.

The $\mathrm{x}$ is the set of estimated variables, $d_{1}$ is an unmeasurable disturbance, $d_{2}$ is a measurable disturbance, $\mathrm{u}$ is the control input of the object, $\mathrm{y}$ is the object measurable output variable, $x^{*}$ is the measured value of calculated value or large sampling interval for possible off-line analysis. Soft measurement modeling is the optimal estimation of the estimated variable $\mathrm{x}$ based on the measured data: $\hat{x}=f\left(d_{2}, u, y, x^{*}, t\right)$ 


\subsection{The Application of Soft Measurement in Fermentation Process Based on Artificial Neural Network.}

The soft measurement modeling method based on artificial neural network [6] is a kind of soft measurement modeling method which has been studied the most and developed rapidly and widely in recent years. Because it can be applied to highly nonlinear and severe uncertainty systems, it provides an effective way to solve the soft measurement problem of complex system process parameters. Artificial neural network (ANN) -- with excellent information processing characteristics: without the prior knowledge of objects, the data can be modeled directly according to the input and output data of the object. The unique non-traditional expression and the inherent learning ability have great potential to solve the highly nonlinear aspect.

\subsubsection{Feasibility Analysis of Soft Measurement Modeling Using RBF Neural Network.}

RBF neural network has gradually replaced BP neural network with its excellent performance and has become a major research hotspot. The RBF neural network is used for soft measurement modeling with the following character:

RBF neural network is a kind of forward network with good performance. There is no local optimal problem of BP network learning in nonlinear modeling, and the learning complexity is low and the learning efficiency is high. The online learning method can completely enable RBF network to track the system which is controlled and detected in real time.

The RBF neural network can realize the universality of the soft measurement model.

\subsubsection{Design and Analysis of RBF Neural Network.}

(1) Determination of RBF network model.

The RBF (RadialBasisFunetion) neural network consists of three layers [7], as shown in figure 2. Input layer nodes only ethanol concentration input signals to the hidden layer, hidden layer nodes composed of radial effect function such as gaussian function, the output layer nodes is usually a simple linear function. The role of hidden layer nodes function (function) of the input signal in the local response, that is, only when the input signal is closer to the middle of the basis function of scope, the output of the hidden layer nodes will produce larger, thus this kind of network with local approximation ability, so the radial basis function network is also known as local field network perception [8].

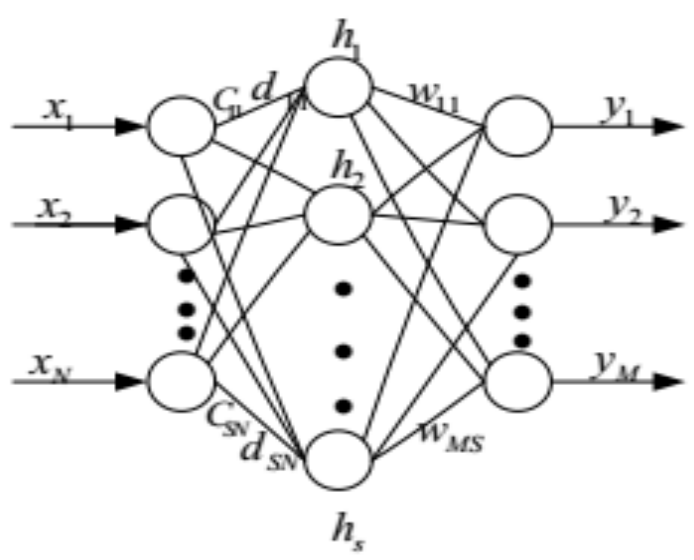

Fig. 3 RBF network structure diagram.

(2)The form of the basis function.

As a basis function, there are several types:

$$
\begin{gathered}
f(x)=\exp ^{-\left(\frac{x}{\delta}\right)^{2}} \\
f(x)=\frac{1}{\left(\delta^{2}+x^{2}\right)}, \quad \alpha>0 \\
f(x)=\left(\delta^{2}+x^{2}\right)^{\beta}, \quad \alpha<\beta<1
\end{gathered}
$$


The above functions are all radial symmetric, although there are various radial basis functions [9], but the most commonly used is Gaussian Kernel funetion, as shown in the following formula:

$$
R_{i}(x)=\exp \left[-\frac{\left\|x-c_{i}\right\|^{2}}{2 \sigma_{i}^{2}}\right], i=1,2, \ldots, \mathrm{m}
$$

Where $\mathrm{x}$ is the $\mathrm{n}$-dimensional input vector: $c_{i}$ is the center of the $\mathrm{i}$ basis function, the vector that has the same dimension as $\mathrm{x}: \sigma_{i}$ is the ith perceptible variable (optional parameter) that determines the width of the base function around the center point; $M$ is the number of perceptive units. $\left\|x-c_{i}\right\|$ is the norm of $x-c_{i}$, which usually represents the Euclidean distance between $\mathrm{x}$ and $c_{i}, R_{i}(x)$ has a unique maximum at $c_{i}$, and as $\left\|x-c_{i}\right\|$ goes up, $R_{i}(x)$ goes down to zero. For a given input: $x \in R^{n}$, only a small fraction of the value of the center near $\mathrm{x}$ is activated.

As can be seen from the RBF structure diagram, the input layer realizes the nonlinear mapping of $x \rightarrow R_{i}(x)$, and the output layer realizes the linear mapping from $R_{i}(x)$ to $y_{k}$, namely:

$$
y_{k}=\sum_{i=1}^{m} w_{i k} R_{i}(x), k=1,2, \ldots, p
$$

Where $\mathrm{p}$ is the number of nodes in the output layer.

Because $R_{i}(x)$ for gaussian function, thus for any $\mathrm{x}, R_{i}(x)>0$, thus lose the advantages of local cumulative values, as a matter of fact, when $\mathrm{x}$ away from $c_{i}, R_{i}(x)$ is very small, thus it can be as 0 , so only $R_{i}(x)$ is greater than a certain value (e.g. 05) to the corresponding weights are modified. After this treatment, RBF network. The network also has the advantages of local approximation to the fast convergence speed. At the same time, this approximation can overcome the shortcomings of the gaussian function without compactness to some extent.

The gauss function [10] used above has the following advantages:

1) The representation is simple, even for multivariable input, it does not add too much complexity;

2) Radial symmetry;

3) Good smoothness, the existence of any derivative;

4) Because the basis function is simple and analytical, it is convenient for theoretical analysis.

(3)RBF learning process.

The learning process of RBF is divided into two stages. In the first stage, according to all input samples, the central value $c_{i}$ and $\sigma_{i}$ of the gaussian function of each node of the hidden layer are determined. In the second stage, after determining the parameters of the hidden layer, the weight $w_{i}$ of the output layer is obtained by using the least squares principle according to the sample. Experimental measurement errors are shown as shown in table 2.

Table 2.Neural network precision table

\begin{tabular}{|c|c|c|}
\hline Neural network & Prediction error & Actual error \\
\hline Error value & 0.03325 & 0.03379 \\
\hline
\end{tabular}

\section{Conclusion}

According to the complexity, highly nonlinear and time variant mechanism of fermentation process, this paper established a soft measurement model based on RBF neural network, which has the advantages of simple structure, strong self-learning ability, can capture the nonlinear characteristics of the process, although the system has begun to model, but because the system is relatively large, but also to test and further tests. 


\section{References}

[1]. Tong Hao, Application of soft sensing technology in biological fermentation process, master's degree thesis of Zhejiang University, 2004.

[2]. Rong Gang, Jin Xiaomin, Wang Shuqing, Advanced control technology and application: Third, soft sensing technology and its application, chemical automation and instrument, 1999, 26(4), 70-72.

[3]. Wang Xudong, Shao Huihe, Neural network modeling and soft measurement technology, chemical automation and instrumentation,1996,23(2),28-31.

[4]. Sonnleitner B, Fiechter A, Impacts of automated bioprocess systems on mondern biological research, Advances in Biochemical Engineering Biotechnology, New york,1992,46,142 -159.

[5]. Wang Yijun, Fan Yu. [J]. Progress in biochemistry and Biophysics,2000,27(4):

[6]. 387-390.

[7]. Xu Yong Yi, Neural network pattern recognition and its implementation. Beijing: Beijing Electronic Industry Press, 1996.6

[8]. P1ggio T, Girosi F.A Theory of Networks for Approximation and Learning. A I Mem No1140,Artifieial Intelligence Laboratory Massaehusetts Institute of Teehnology, Cambridge Mass, 1989

[9]. Moody J,Darken C, Fast Learning in Networks of Loeally -tuned Proeessing Units, Neural Computation,1989,(1):281-294

[10]. ChenS, Billings S A, Grant P M.Reeursive Hybride Algorithm for Nonlinear System Identifieation Using Radial Basis Funetion Networks, Int F Control,1992,55(5):1051-1070 